\title{
Effect of isocenter deviation on volume modulated arc therapy plan results and gamma passing rate in the treatment of cervical cancer
}

\author{
Yuan Yuan ${ }^{1}$, Xiao-Bin Chang ${ }^{2} \wedge$, Li-Juan Hu${ }^{1}$, Guo-Qing Wang ${ }^{1}$, Yan-Jun Xue ${ }^{3}$ \\ ${ }^{1}$ Department of Gynaecological Oncology, Shaanxi Provincial Tumor Hospital, Affiliated Hospital of Xi'an Jiaotong University Health Science \\ Center, Xi'an, China; ${ }^{2}$ Department of Radiation Oncology, Shaanxi Provincial Tumor Hospital, Affiliated Hospital of Xi'an Jiaotong University \\ Health Science Center, Xi'an, China; ${ }^{3}$ Financial Department, Shaanxi Provincial Tumor Hospital, Affiliated Hospital of Xi'an Jiaotong University \\ Health Science Center, Xi'an, China \\ Contributions: (I) Conception and design: XB Chang; (II) Administrative support: GQ Wang; (III) Provision of study materials or patients: Y Yuan; (IV) \\ Collection and assembly of data: Y Yuan; (V) Data analysis and interpretation: LJ Hu; (VI) Manuscript writing: All authors; (VII) Final approval of \\ manuscript: All authors. \\ Correspondence to: Guo-Qing Wang. Department of Gynaecological Oncology, Shaanxi Provincial Tumor Hospital, Affiliated Hospital of Xi'an \\ Jiaotong University Health Science Center, Xi'an 710061, China. Email: wgq5668@163.com.
}

Background: Isocenter deviation, often induced by small displacements of both the device and the patient, is a common error seen in radiotherapy. In this study, we investigated the impact of isocenter deviation on the results of the volumetric modulated arc therapy (VMAT) plan and dosimetric verification gamma passing rate in the treatment of cervical cancer.

Methods: The clinical data of 15 patients with cervical cancer who were treated with VMAT were retrospectively collected and analyzed. In this study, the isocenter site modification method was adopted. The VMAT plan with isocenter deviation adjustment was set as the experimental group, while the original plan was set as the control group. The impact of isocenter deviation on the results of the VMAT plan and dosimetric verification gamma passing rate was analyzed. Applying gamma analysis with different test criterions, the impact of isocenter deviation on the gamma passing rates was evaluated, and the sensitivity of different test criterions in identifying isocenter deviation was also analyzed.

Results: There was a significant difference in the average dose in the target area between experimental group and control group $(\mathrm{P}<0.05)$. In organs at risk $(\mathrm{OAR})$ terms, isocenter deviations also caused significant differences in dose parameters between the two groups. Except that there was no significant difference in the rectal V40 between two groups when the isocenter deviation was greater than $3 \mathrm{~mm}$ in the y axis direction. With the increase in the isocenter deviation, there was a trend towards decreased gamma passing rates with different analysis criterions in the experimental group. The $2 \mathrm{~mm} / 2 \%$ standard had the highest sensitivity for identifying isocenter deviation.

Conclusions: Isocenter deviation has significant effects on the results of the volume rotation intensity modulation plan and dosimetric verification gamma passing rates in the treatment of cervical cancer. When the isocenter deviation was less than $3 \mathrm{~mm}$, a higher gamma passing rate (>90\%) could also be obtained under the condition of the $3 \mathrm{~mm} / 3 \%$ test criterions. It is recommended that the $2 \mathrm{~mm} / 2 \%$ test standard should be utilized in clinical practice.

Keywords: Isocenter; cervical cancer; volume rotation intensity modulation; Delta4; $\gamma$ analysis

\footnotetext{
^ ORCID: 0000-0003-0038-0464.
} 
Submitted May 12, 2021. Accepted for publication Sep 07, 2021.

doi: $10.21037 /$ tcr-21-1514

View this article at: https://dx.doi.org/10.21037/tcr-21-1514

\section{Introduction}

Radiotherapy has become the major treatment for cervical cancer. Concurrent radiochemotherapy is routinely performed in patients with advanced cervical cancer, and has been shown to improve the prognosis of these patients (1). For those with a high risk of tumor recurrence after surgery, preventive pelvic radiotherapy could decrease the risk of local recurrence by $41 \%$ (2). Since 1995 when the concept of volumetric modulated arc therapy (VMAT) was first put forward (3), this approach, which has a high target conformity index, good target dose distribution, and short therapeutic time $(4,5)$, has been established for the treatment of cervical cancer (6-8).

VMAT is a novel delivery method of intensitymodulated radiotherapy (IMRT). It is capable of delivering highly conformal dose distributions through concomitant continuous gantry rotation, dynamic beam modulation and variable dose rate. However, VMAT places greater demands on the therapeutic accuracy of the electron linear accelerator, including the gantry speed, the stability of the dose rate, the positioning accuracy of the gantry and multileaf collimator (MLC), and the accuracy of the isocenter localization. The uncertainty of these parameters can lead to a mismatch of the prescription dose and the actual dose. In the process of radiotherapy, isocenter deviations, including those induced by the movement of the bed, the laser indication light, and the gravity of the gantry head, are the most common system errors. Thus, identifying the isocenter deviation is pivotal for the results of the volume rotation intensity modulation treatment plan. The 50th report released by the International Commission on Radiation Units and Measurements (ICRU) pointed out that a higher deviation ( $>5 \%$ ) would increase the risk of the occurrence of radiotherapy-related complications and uncontrolled growth of the primary tumor (9). The 40th report released by the American Association of Physicists in Medicine (AAPM) suggested that independent examination of monitor units should be performed before radiotherapy, and the traditional manual examination for single point dosage was unsuitable for VMAT owing to the complexity of the therapeutic plan (10-12).

Nowadays, the combination of virtual water and 2D or $3 \mathrm{D}$ dose detector matrices is usually used. Gamma analysis is used to determine the correlation of the dose of the treatment planning system with that of the electron linear accelerator $(13,14)$. Dosimetric verification gamma passing rate is used to determine whether treatment plan can be used for clinical treatment. Gamma index with 3\% dose difference and $3 \mathrm{~mm}$ dose-to-distance criteria are most commonly used by physicists in pre-treatment IMRT and VMAT dosimetric verification as reported in the AAPM Task Group 119. It is performed by exporting the Digital Imaging and Communications in Medicine (DICOM) files. Then, contrastive analysis of the actual dose distribution measured by the detector matrices is performed, and the difference between the prescription dose and the actual dose is measured, thus determining whether the uncertainty of the prescription dose for radiotherapy is acceptable in clinical practice. In 2019, Oulhouq et al. introduced error and analyzed the effects of rotation of the small rack and big rack, as well as isocenter deviation, on the results of the radiotherapy plan via the combination of 6 megavolt (MV) photon and 12 megaelectronvolt (Mev) electron beams. They observed that an isocenter deviation of $1 \mathrm{~mm}$, instead of rotation of the small rack within $0.5^{\circ}$, significantly influenced the results of the radiotherapy plan (15). Heilemann et al. analyzed the effect of setup error of the MLC on the results of the radiotherapy plan and gamma passing rate via $6 \mathrm{MV}$ photon beams. They observed that the setup error of the MLC significantly influenced the results of the radiotherapy treatment plan, and the routine gamma analysis standard $(3 \mathrm{~mm} / 3 \%)$ failed to detect a minor setup error of the MLC, thereby demanding stricter analysis standards (16). Based on these studies, we further analyzed the effect of isocenter deviation on the results of the radiotherapy plan in the treatment of cervical cancer. Differing from previous study protocols, the $6 \mathrm{MV}$ flattening filter free (FFF) mode was utilized for radiotherapy, and isocenter deviation was introduced from both the $\mathrm{X}$ and $\mathrm{Y}$ axes. The effects of isocenter deviation of cervical cancer radiotherapy, as well as the sensitivity of different analysis criterions of the gamma passing rate in identifying isocenter deviations, were analyzed.

We present the following article in accordance with the MDAR reporting checklist (available at https://dx.doi. 
org/10.21037/tcr-21-1514).

\section{Methods}

\section{Clinical data and the design of the radiotherapy plan}

\section{Clinical data}

A total of 15 patients admitted to our hospital between January 2019 and July 2020 who received cervical cancer (stage IIA-IIIA) radiotherapy were included in this retrospective cohort study. The median age of these patients was 52 [30-65] years old. Since this is a retrospective study, only clinical data were collected, with no interventions on previous therapeutic protocols and no potential risk during the treatment. Patient privacy was protected during the study. All procedures performed in this study involving human participants were in accordance with the Declaration of Helsinki (as revised in 2013). The study was approved by ethics board of Shaanxi Provincial Tumor Hospital (No.: 201900033). All patients had no radiotherapy contraindications and provided informed consent for radiotherapy before the treatment.

\section{CT simulated localization and target volume delineation}

A supine position with both hands held above the head and both legs kept together was used and the patients were fixed by a thermoplastic body mask technique during CT (Brilliance CT Big Bore, Philips, the Netherlands) simulation. The scanned area ranged from the upper margin of the first lumbar vertebra to $5 \mathrm{~cm}$ below the ischial tuberosity, with the scanning parameter of $120 \mathrm{KV}$, $300 \mathrm{~mA}$ and a scanning thickness of $3 \mathrm{~mm}$ (axis scan). Then, the acquired CT image sequences were transmitted to the radiotherapy treatment planning system Eclipse ${ }^{\circledR}$ TPS (version 13.16; Varian Medical Systems, Palo Alto, CA, USA) in the format of DICOM. The radiotherapy treatment plan was determined according to the expert consensus of target volume delineation for postoperative radiotherapy of cervical cancer released by the RTOG and the target volume delineation for lymphatic drainage area suggested by Taylor et al. $(17,18)$. The clinical target volume (CTV) included the stump of the vagina, paravaginal soft tissue, and the pelvic lymphatic drainage area (including common iliac lymph nodes, external iliac lymph nodes, internal iliac lymph nodes, obturator lymph nodes, and presacral lymph nodes), which ranged from the 4th/5th lumbar vertebra to the lower margin of the obturator. The planning target volumes (PTV) were determined by extension of $8 \mathrm{~mm}$ in the up-down, left-right, and anterior directions and $5 \mathrm{~mm}$ in the posterior direction. Organs at risk (OAR) included the spinal cord, rectum, bladder, intestine, and bilateral heads of the femur.

\section{The design of radiotherapy planning}

The TPS was utilized for treatment plan, and VMAT was used for the treatment of all patients. TrueBeam (Trubeam1697, Varian Medical Systems, Palo Alto, CA, USA) electron linear accelerator, in the $6 \mathrm{MV}$ FFF mode, was used in this study. The total prescribed dose was $50 \mathrm{~Gy}$, with $2 \mathrm{~Gy}$ in each fraction. A double arc design, with a clockwise arc of $181^{\circ}-179^{\circ}$ and anticlockwise arc of $179^{\circ}-181^{\circ}$, was used. AcurosXB (version 13.6) was used to calculate the dose. Additionally, $100 \%$ of the prescribed dose should be guaranteed to cover $95 \%$ of the PTV, with the highest dose less than $107 \%$ of the prescribed dose. The Dmax for the spinal cord, the rectum/bladder, the intestine, and the bilateral heads of the femur were $40 \mathrm{~Gy}, \mathrm{~V} 40<50 \%$, $\mathrm{V} 35<30 \%$, and V $50<5 \%$, respectively.

\section{Experimental design and gamma analysis}

\section{Experimental design}

The volume rotation intensity modulation plan which introduced the isocenter deviation was set as the experimental group, while the original plan was set as the control group. The method of introducing error in the volume rotation intensity modulation plan is described in Figure $1 A$. With the isocenter as the coordinate point, four different radiotherapy plans were made after introducing errors of $1,2,3$, and $5 \mathrm{~mm}$ in the $\mathrm{X}$ axis, and another four different radiotherapy plans were made after introducing errors of $1,2,3$, and $5 \mathrm{~mm}$ in the $\mathrm{Y}$ axis. The dose parameters of PTV and OAR were calculated in the control group and the experimental group. The Delta4 diode array phantom (Scandidos, Uppsala, Sweden) was used to verify the dose of the radiotherapy plan in the control group. Before verification, strict quality control was performed in the electron linear accelerator to satisfy the therapeutic requirement. The method to verify the dose of the radiotherapy plan is described in Figure $1 B$. The isocenter deviation was introduced based on the isocenter of the Delta4 phantom. The two isocenters were moved in both the $\mathrm{X}$ and $\mathrm{Y}$ axes via moving the therapeutic bed. Errors of 1, 2, 3, and $5 \mathrm{~mm}$ were introduced in the $\mathrm{X}$ axis. Then, four gamma passing rates were obtained via different gamma analysis criterions of $3 \mathrm{~mm} / 3 \%, 2 \mathrm{~mm} / 2 \%$, and $1 \mathrm{~mm} / 1 \%$ in the 

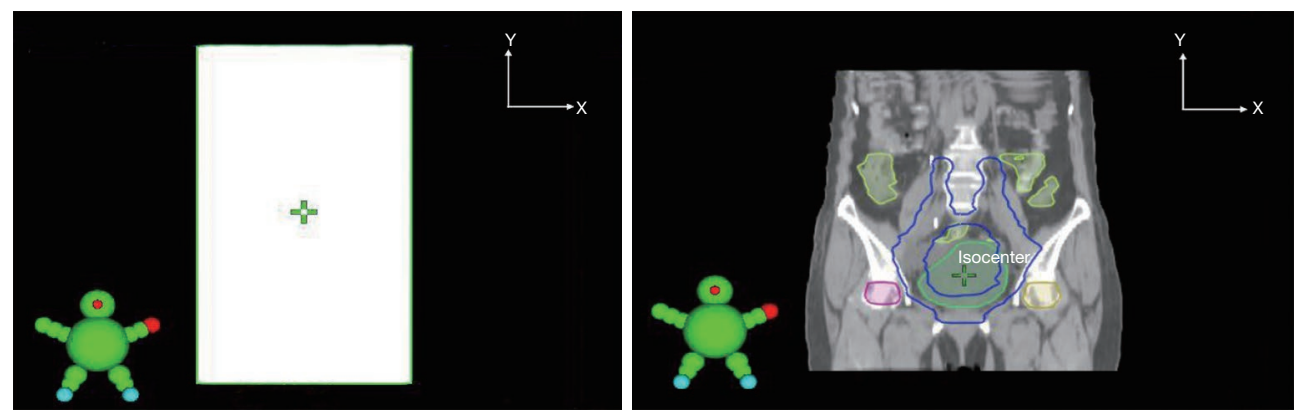

Figure 1 Schematic diagram showing the introduction of isocenter deviation in the coronal plane of CT and the Delta4 model. The blue area is PTV, the dark green area is the bladder volume and the light green area is the small intestine volume. The green crosshairs indicate the position of the isocenter. (A). Patient coronal section; (B) Delta4 phantom coronal section. PTV, planning target volume.

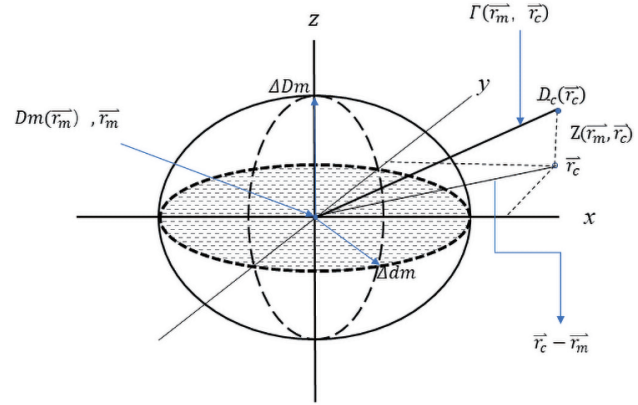

Figure 2 The schematic diagram for gamma index analysis. $\delta\left(\vec{r}_{m}, \vec{r}_{c}\right)$ denotes the discrepancy of dose between the counted point and the measurement point. $\overline{r_{c}}-\overline{r_{m}}$ denotes the discrepancy of location between the counted point and the measurement point.

verification of the dose of the radiotherapy plan. In addition, errors of $1,2,3$, and $5 \mathrm{~mm}$ were introduced in the $\mathrm{Y}$ axis. Four gamma passing rates were obtained via different gamma analysis criterions of $3 \mathrm{~mm} / 3 \%, 2 \mathrm{~mm} / 2 \%$, and $1 \mathrm{~mm} / 1 \%$ in the verification of the dose of the radiotherapy plan.

\section{Gamma analysis}

The radiotherapy plan was transferred into the Delta4 semiconductor array phantom, and the verification plan for dose distribution in the phantom was then generated. The electron linear accelerator was used to implement the verification plan, and actual dose distribution was measured by the Delta 4 semiconductor array phantom. ScandiDos software (version 2015 June) was used to obtain the gamma passing rate. The gamma index, which combined two standards including dose difference and distance-toagreement, was used to examine the consistency of the doses. As recommended by the AAPM TG-119 report, if the gamma passing rate is higher than $90 \%$ under the condition of $3 \mathrm{~mm} / 3 \%$, the radiotherapeutic plan can be used for clinical treatment (19). Nowadays, the $3 \mathrm{~mm} / 3 \%$ criterions is routinely used in clinical practice. According to the theory put forward by Low et al., the principle for gamma analysis is described in Figure 2 (20). In the spheroid, $\Delta \mathrm{Dm}$ is the standard for analyzing the gamma dose deviation, while $\Delta \mathrm{dm}$ is the standard for analyzing the gamma location consistency. For the dosimetric verification, the clinically acceptable range was indicated by the equation in the spheroid as the surface representing the acceptance criterion. The evaluation presented is for a single measurement point $r_{m}$, lying at the origin of the figure, and for clinical evaluations, the comparisons are repeated for all measurement points. The equation defining the surface is

$$
1=\sqrt{\frac{r^{2}\left(r_{m}, r\right)}{\Delta d^{2}{ }_{M}}}+\frac{z^{2}\left(r_{m}, r\right)}{\Delta D^{2}{ }_{M}}
$$

Where $r\left(r_{m}, r\right)=\left|r-r_{m}\right|$, and $z\left(r_{m}, r\right)=D(r)-D_{m}\left(r_{m}\right)$ is dose difference at the position $\mathrm{r}_{\mathrm{m}}$.

Figure 2 depicts the schematic diagram for gamma index analysis. Two of the axis ( $\mathrm{X}$ and $\mathrm{Y}$ ) represent the spatial location $r_{c}$ of calculated distribution relative to the measured point. The third axis $(z)$ represents the difference between the measured $D_{m}\left(r_{m}\right)$ and calculated $D_{c}\left(r_{c}\right)$ doses. If any portion of the $D_{c}\left(r_{c}\right)$ surface intersects the ellipsoid defined by Eq. [1], the calculation passes at $r_{m}$. In the $r_{c}-r_{m}$ plane allows for a more general comparison between calculation and measurement than does the traditional composite evaluation. The quantity on the right-hand side of Eq. [1] can be used to identify a quality index $\gamma$ at each point in the 
Table 1 The effect of isocenter deviation on the exposure dose of the PTV

\begin{tabular}{lcc}
\hline Deviation & Dmean (cGy) & $P$ \\
\hline$X=0, Y=0$ & $5,195.83 \pm 31.00$ & - \\
$X=1 \mathrm{~mm}$ & $5,367.89 \pm 48.82$ & 0.00 \\
$X=2 \mathrm{~mm}$ & $5,255.67 \pm 34.33$ & 0.00 \\
$X=3 \mathrm{~mm}$ & $5,222.16 \pm 31.41$ & 0.00 \\
$X=5 \mathrm{~mm}$ & $5,201.89 \pm 30.47$ & 0.02 \\
$Y=1 \mathrm{~mm}$ & $5,257.75 \pm 28.87$ & 0.00 \\
$Y=2 \mathrm{~mm}$ & $5,225.60 \pm 31.38$ & 0.00 \\
$Y=3 \mathrm{~mm}$ & $5,204.88 \pm 31.64$ & 0.00 \\
$Y=5 \mathrm{~mm}$ & $5,195.83 \pm 31.00$ & 0.00 \\
\hline
\end{tabular}

Deviation denotes the isocenter deviation. $X$ represents the $X$ axis, while $Y$ represents the $Y$ axis. Dmean denotes the average dose of the target area of the PTV. PTV, planning target volumes.

evaluation plane $r_{c}-r_{m}$ for the measurement point $r_{m}$.

$$
\gamma\left(r_{m}\right)=\min \left\{\Gamma\left(r_{m}, r_{c}\right)\right\} \forall\left\{r_{c}\right\}
$$

Where $\Gamma\left(r_{m}, r_{c}\right)=\sqrt{\frac{r^{2}\left(r_{m}, r_{c}\right)}{\Delta d^{2}{ }_{M}}}+\frac{z^{2}\left(r_{m}, r_{c}\right)}{\Delta D^{2}{ }_{M}}, r\left(r_{m}, r_{c}\right)=\left|r_{c}-r_{m}\right|$,

and $z\left(r_{m}, r_{c}\right)=D_{c}\left(r_{c}\right)-D_{m}\left(r_{m}\right)$.

For the gamma index analysis between dose values on the calculated and measured distributions, if the gamma value of a point was higher than 1 , the gamma analysis failed; otherwise it succeeded. For example, the routine standard for the clinical radiotherapy plan is $\Delta \mathrm{dm}=3 \mathrm{~mm}$, $\Delta \mathrm{Dm}=3 \%$. If the $\gamma\left(\mathrm{r}_{\mathrm{m}}\right)$ is less than 1 for more than $90 \%$ of the examined points, the validation for the therapeutic dose passes.

\section{Statistical analysis}

Quantitative data with a non-normal distribution were expressed as mean \pm standard deviation (SD). Normality tests for PTV and OAR data were performed by the Shapiro-Wilk test, and all data met the normal distribution $(\mathrm{P}>0.05)$. Paired $t$-tests were performed with SPSS software (version 22.0, IBM Corp., USA). $\mathrm{P}<0.05$ was considered to indicate statistical significance. The sensitivity of different standards of gamma analysis in identifying isocenter deviations was also measured. Dose-volume histograms were plotted via Origin2016 software, and the trend of isocenter deviations with different gamma analysis standards ( $3 \mathrm{~mm} / 3 \%, 2 \mathrm{~mm} / 2 \%, 1 \mathrm{~mm} / 1 \%$ ) was observed. To analyze the rate of change of the gamma passing rate in the condition of different isocenter deviations compared to the condition of no isocenter deviation, the trend line was also plotted, and the sensitivity of different standards of gamma analysis in identifying isocenter deviations was also measured.

\section{Results}

\section{The effect of isocenter deviation on the mean dose of the PTV}

Paired $t$-tests were performed to compare the average exposure dose in the target area of the PTV (Table 1). The results showed that isocenter deviations in both the $\mathrm{X}$ and $\mathrm{Y}$ axes could affect the Dmean (the average dose of PTV) of the PTV of the therapeutic plan in the experimental group $(\mathrm{P}<0.05)$. Along with the increase in the isocenter deviation, the average dose in the target area of the PTV gradually decreased.

Paired $t$-tests were performed to compare the parameters of the OAR (Table 2). When the isocenter deviation was greater than $2 \mathrm{~mm}$ in the $\mathrm{X}$ and $\mathrm{Y}$ axes, a significant difference in bladder V40 (the percent of volume included by $40 \mathrm{~Gy}$ ) between the two groups was found $(\mathrm{P}<0.05)$. Along with the increase in the isocenter deviation in both the $\mathrm{X}$ and $\mathrm{Y}$ axes, the average bladder $\mathrm{V} 40$ increased. In the $\mathrm{X}$ axis, a significant difference in the rectum V40 was found between the control group and the experimental group $(\mathrm{P}<0.05)$. In the $\mathrm{Y}$ axis, no significant difference in the rectum V40 was observed when the isocenter deviation was greater than $2 \mathrm{~mm}(\mathrm{P}>0.05)$. The isocenter deviation in the $\mathrm{X}$ axis had an obvious impact on the exposure dose of the rectum. When the isocenter deviation was greater than $2 \mathrm{~mm}$, it had an obvious impact on the distribution of the exposure dose of the intestine $(\mathrm{P}<0.05)$. In the $\mathrm{Y}$ axis, all the isocenter deviations in the experimental group had significant effects on the distribution of the exposure dose of the intestine $(\mathrm{P}<0.05)$. Along with the increase in the isocenter deviation, the average intestine V40 gradually decreased, and the isocenter deviation in the $\mathrm{Y}$ axis had a significant impact on the exposure dose of the intestine. 
Table 2 The effect of isocenter deviation on the exposure dose of the OAR

\begin{tabular}{|c|c|c|c|c|c|c|}
\hline Deviation & \multicolumn{2}{|c|}{ Bladder V40 } & \multicolumn{2}{|c|}{ Rectum V40 } & \multicolumn{2}{|c|}{ Intestine V30 } \\
\hline$X=0, Y=0$ & $39.53 \pm 10.90$ & - & $39.53 \pm 12.46$ & - & $6.01 \pm 5.01$ & - \\
\hline $\mathrm{X}=1 \mathrm{~mm}$ & $39.54 \pm 10.93$ & 0.94 & $40.00 \pm 12.34$ & 0.04 & $6.08 \pm 5.03$ & 0.11 \\
\hline$X=2 \mathrm{~mm}$ & $39.83 \pm 10.90$ & 0.62 & $41.04 \pm 12.26$ & 0.00 & $6.24 \pm 5.06$ & 0.06 \\
\hline$X=5 \mathrm{~mm}$ & $42.44 \pm 11.17$ & 0.01 & $47.34 \pm 12.20$ & 0.00 & $7.25 \pm 5.29$ & 0.01 \\
\hline $\mathrm{Y}=1 \mathrm{~mm}$ & $39.86 \pm 10.85$ & 0.22 & $38.70 \pm 12.27$ & 0.02 & $6.13 \pm 5.06$ & 0.00 \\
\hline $\mathrm{Y}=2 \mathrm{~mm}$ & $40.45 \pm 10.91$ & 0.13 & $38.29 \pm 12.15$ & 0.00 & $6.32 \pm 5.09$ & 0.00 \\
\hline $\mathrm{Y}=3 \mathrm{~mm}$ & $40.96 \pm 11.09$ & 0.09 & $38.93 \pm 12.16$ & 0.33 & $6.62 \pm 5.10$ & 0.00 \\
\hline
\end{tabular}

Bladder V40 denotes the volume included by bladder 40 Gy. Rectum V40 denotes the volume included by rectum 40 Gy. Intestine V30 denotes the volume included by intestine $30 \mathrm{~Gy}$. SD, standard deviation; OAR, organs at risk.

Table 3 The change of the gamma passing rate in different isocenter deviations in the radiotherapy plans

\begin{tabular}{|c|c|c|c|c|c|c|}
\hline Deviation & \multicolumn{2}{|c|}{3 mm/3\% } & \multicolumn{2}{|c|}{2 mm/2\% } & \multicolumn{2}{|c|}{$1 \mathrm{~mm} / 1 \%$} \\
\hline$X=0, Y=0$ & $95.17 \pm 2.59$ & - & $81.80 \pm 7.03$ & - & $48.70 \pm 9.52$ & - \\
\hline $\mathrm{X}=1 \mathrm{~mm}$ & $94.61 \pm 2.45$ & 0.025 & $80.80 \pm 6.76$ & 0.025 & $47.30 \pm 9.03$ & 0.050 \\
\hline $\mathrm{X}=2 \mathrm{~mm}$ & $92.31 \pm 2.95$ & 0.001 & $76.20 \pm 7.81$ & 0.001 & $42.30 \pm 9.45$ & 0.002 \\
\hline$X=5 \mathrm{~mm}$ & $67.63 \pm 9.43$ & 0.000 & $41.40 \pm 11.26$ & 0.000 & $15.50 \pm 7.45$ & 0.000 \\
\hline $\mathrm{Y}=1 \mathrm{~mm}$ & $94.30 \pm 2.70$ & 0.002 & $80.10 \pm 7.49$ & 0.003 & $46.50 \pm 9.74$ & 0.001 \\
\hline $\mathrm{Y}=2 \mathrm{~mm}$ & $91.71 \pm 3.50$ & 0.002 & $74.90 \pm 9.10$ & 0.000 & $40.90 \pm 10.01$ & 0.000 \\
\hline $\mathrm{Y}=3 \mathrm{~mm}$ & $87.14 \pm 5.67$ & 0.001 & $65.60 \pm 11.77$ & 0.000 & $32.10 \pm 9.47$ & 0.000 \\
\hline
\end{tabular}

$3 \mathrm{~mm} / 3 \%, 2 \mathrm{~mm} / 2 \%$, and $1 \mathrm{~mm} / 1 \%$ are different detection standards for gamma analyses. SD, standard deviation.

\section{The effect of isocenter deviation on gamma passing rates}

Under almost all the test standards, the isocenter deviation of $1 \mathrm{~mm}$ had a significant impact on the gamma passing rate $(\mathrm{P}<0.05)$. However, under the test standard of $1 \mathrm{~mm} / 1 \%$, no significant difference was observed in the gamma passing rate between the isocenter deviation of $1 \mathrm{~mm}$ and no isocenter deviation $(\mathrm{P}=0.05)$ (Table 3).

As indicated in the histogram (Figure 3), along with the increase in the isocenter deviation, the gamma passing rates gradually decreased. A similar trend was seen when other test standards were used. Under the condition of the
$3 \mathrm{~mm} / 3 \%$ criterions, a gamma passing rate higher than $90 \%$ was deemed to meet the standard. When the isocenter deviation was greater than $2 \mathrm{~mm}$, the gamma passing rate fell below $90 \%$.

The rate of change of the gamma passing rate in radiotherapy planning under the condition of different isocenter deviations was calculated based on the gamma passing rate in the control group. The point plot concerning the rate of change between the isocenter deviation and gamma passing rate is shown in Figure 4. Along with the increase in the error of the isocenter deviations, the rate 

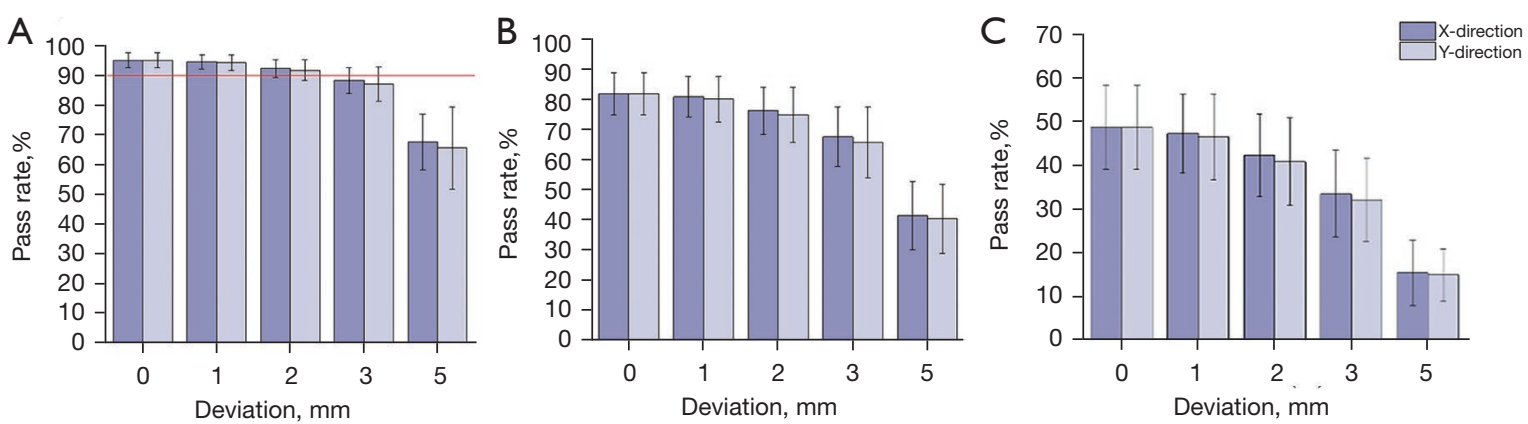

Figure 3 Histograms showing the analysis of isocenter deviations in the $\mathrm{X}$ and $\mathrm{Y}$ axes via different criterions. The $\mathrm{X}$ axis denotes the isocenter deviation. The $\mathrm{Y}$ axis denotes the gamma passing rate. (A) $3 \mathrm{~mm} / 3 \%$ criterions; (B) $2 \mathrm{~mm} / 2 \%$ criterions; (C) $1 \mathrm{~mm} / 1 \% \mathrm{criterions}$.
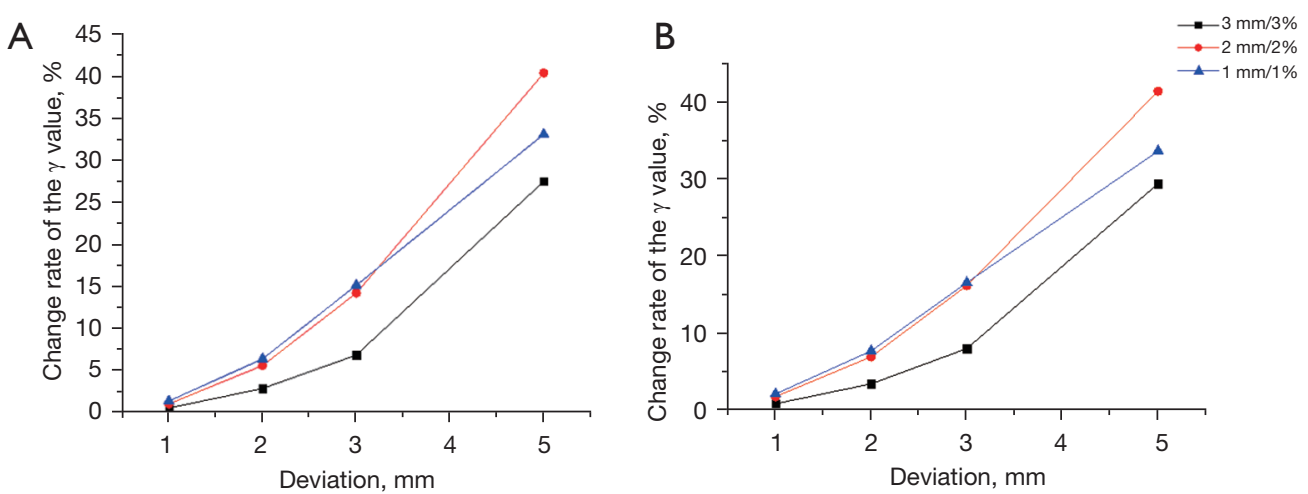

Figure 4 Line graphs showing the relationships between the isocenter deviations and gamma passing rates. (A) Relationship between the change rate of the $\gamma$ value and deviation in the $\mathrm{X}$ direction; (B) relationship between the change rate of the $\gamma$ value and deviation in the $\mathrm{Y}$ direction.

of change of the gamma passing rates gradually increased under the condition of different test standards. Along with the change in the isocenter deviation in the $\mathrm{X}$ or $\mathrm{Y}$ axes, the rate of change of the gamma passing rate had similar changing trends. According to the slope of the curve, we observed that the test criterions of $2 \mathrm{~mm} / 2 \%$ and $1 \mathrm{~mm} / 1 \%$ had a stronger sensitivity than the $3 \mathrm{~mm} / 3 \%$ criterions for identifying the isocenter deviation when the isocenter deviation was less than $3 \mathrm{~mm}$.

\section{Discussion}

Isocenter deviation is the most common error during the process of radiotherapy for cervical cancer, which is caused by changes in the position of the patients, the mechanical accuracy of the electron linear accelerator, the effect of gravity on the rack of the electron linear accelerator, the indicator error of the isocenter laser, and the movement of the patients. Hence, the analysis of the uncertainty in the radiotherapy plan caused by these errors has important clinical implications. In this study, isocenter deviations in both the $\mathrm{X}$ and $\mathrm{Y}$ axes were introduced and we observed that the isocenter deviation could lead to changes in the exposure dose of the patients. An isocenter deviation greater than $1 \mathrm{~mm}$ could induce significant change in the distribution of the radiotherapy plan dose, which was in accordance with a previous study by Oulhouq et al. (15). From the aspect of the therapeutic target, isocenter deviation has a significant influence on the average dose of the PTV. The average dose gradually decreases along with the increase in isocenter deviation. When the isocenter deviation gradually increases, radiotherapy gradually loses complete coverage of the target area, thus leading to a decrease in the average dose in the target area. From the aspect of injury to adjacent organs, when the isocenter deviation in the $Y$ axis was higher than $3 \mathrm{~mm}$, no significant 
difference was seen in the rectum exposure dose between the control group and the experimental group. We believed that this was due to the relative locations of the PTV and OAR. When the isocenter deviation increases in the positive $\mathrm{X}$ axis or the positive $\mathrm{Y}$ axis, more bladder or rectum volume would be involved in the treatment fields according to the anatomical position of the target area and adjacent organs. Therefore, the parameter of the dose exhibits a monotonic increasing trend. With the increase in isocenter deviation in the $\mathrm{Y}$ axis, there is no significant difference in therapeutic ray accumulated volume in the rectum. According to the above analyses, there are several limitations in the experimental design. Only isocenter deviations in the $\mathrm{X}$ and $\mathrm{Y}$ axes were analyzed, which does not represent all the conditions encountered in actual clinical practice. Thus, further research is urgently needed. The sample size of this study was small, we also need to expand the sample size in the later stage of our research. From the perspective of absolute value of dose difference, when the isocenter deviation in a single direction is greater than $3 \mathrm{~mm}$, the average dose change in the target area is approximately $1-3 \%$, while the change in injury to adjacent organs is approximately $2-5 \%$, which corroborates a previous study concerning the effect of setup error on dose error (21). Taking the isocenter deviation in other directions into account, the difference in the therapeutic plan dose would be larger. Therefore, much more attention should be paid to other factors which may induce isocenter changes in clinical practice. In the routine quality control of clinical devices, significantly more attention should be paid to the electron linear accelerator and the central accuracy of the therapeutic bed. During the period of treatment, techniques such as imaging-guided methods and respiration control are recommended to reduce the positional deviation in the positioning process as well as active individual movements in the treatment process. We recommend cone-beam CT based isocenter position correction for patients at least once a week. Every time we set up the patient's body. we should do it as carefully as possible.

Pre-treatment verification is a key procedure to guarantee the radiotherapy accuracy, and the $3 \mathrm{D}$ verification system based on gamma analysis is widely used in clinical practice $(22,23)$. According to the conclusions in this study, isocenter deviation can obviously influence the gamma passing rate in the radiotherapy plan. Along with the increase in the isocenter deviation, the gamma passing rate robustly decreases. With the verification standard of $3 \mathrm{~mm} / 3 \%$, isocenter deviation less than $3 \mathrm{~mm}$ could also satisfy the passing rate higher than $90 \%$ required in clinic. Based on the $3 \mathrm{~mm} / 3 \%$ analysis standard, most hospitals also use these plans for radiotherapy when the passing rate is higher than $90 \%$. According to the observations in this study, the isocenter deviation of $3 \mathrm{~mm}$ would exert significant effects on the exposure dose in adjacent organs, bringing about great uncertainty in the therapeutic dose. Both Nelms et al. and Zhen et al. observed that even if the gamma passing rate is acceptable, the discrepancy between the actual dose and the estimated dose may exceed the acceptable range in clinical practice $(24,25)$. Hence, only using the $3 \mathrm{~mm} / 3 \%$ analysis standard will overestimate the gamma passing rate in the radiotherapy plan. This study analyzed the sensitivity of different analysis standards in identifying isocenter deviations in terms of the rate of change of the gamma passing rate. The data showed that the $2 \mathrm{~mm} / 2 \%$ analysis standard has better sensitivity when detecting the isocenter deviation, which may be due to the lower tolerance compared to the $3 \mathrm{~mm} / 3 \%$ standard. During the verification of the volume rotation intensity modulation plan via the gamma passing rate in the treatment of cervical cancer, much stricter criterions should be used when the $3 \mathrm{~mm} / 3 \%$ criterions is utilized. Thus, a stricter gamma analysis standard has important implications in clinical practice.

\section{Conclusions}

Isocenter deviations can markedly influence the distribution of the exposure dose in the target area in the treatment of cervical cancer. Greater isocenter deviation will cause insufficiency of the exposure dose in the target area and increase the risk of damage to adjacent organs. When the isocenter deviation is greater than $3 \mathrm{~mm}$, the isocenter deviation should be adjusted. Quality control of the equipment and therapeutic processes should be paid attention to in clinical practice in order to minimize the effect of isocenter deviation. During the verification of the exposure dose in the radiotherapy of cervical cancer via the gamma passing rate, the $2 \mathrm{~mm} / 2 \%$ analysis standard has better sensitivity when detecting the isocenter deviation, whereas much stricter standards should be used when the $3 \mathrm{~mm} / 3 \%$ standard is utilized.

\section{Acknowledgments}

Funding: This study was supported by Shaanxi Province Innovation chain of key industries (Areas of social development), Project Number: 2016KTZDSF-03. 


\section{Footnote}

Reporting Checklist: The authors have completed the MDAR reporting checklist. Available at https://dx.doi. org/10.21037/tcr-21-1514

Data Sharing Statement: Available at https://dx.doi. org/10.21037/tcr-21-1514

Conflicts of Interest: All authors have completed the ICMJE uniform disclosure form (available at https://dx.doi. org/10.21037/tcr-21-1514). The authors have no conflicts of interest to declare.

Ethical Statement: The authors are accountable for all aspects of the work in ensuring that questions related to the accuracy or integrity of any part of the work are appropriately investigated and resolved. All procedures performed in this study involving human participants were in accordance with the Declaration of Helsinki (as revised in 2013). The study was approved by ethics board of Shaanxi Provincial Tumor Hospital (No.: 201900033). All patients had no radiotherapy contraindications and provided informed consent for radiotherapy before the treatment.

Open Access Statement: This is an Open Access article distributed in accordance with the Creative Commons Attribution-NonCommercial-NoDerivs 4.0 International License (CC BY-NC-ND 4.0), which permits the noncommercial replication and distribution of the article with the strict proviso that no changes or edits are made and the original work is properly cited (including links to both the formal publication through the relevant DOI and the license). See: https://creativecommons.org/licenses/by-nc-nd/4.0/.

\section{References}

1. Kuzuya K. Chemoradiotherapy for uterine cancer: current status and perspectives. Int J Clin Oncol 2004;9:458-70.

2. Rotman M, Sedlis A, Piedmonte MR, et al. A phase III randomized trial of postoperative pelvic irradiation in Stage IB cervical carcinoma with poor prognostic features: follow-up of a gynecologic oncology group study. Int J Radiat Oncol Biol Phys 2006;65:169-76.

3. Yu CX. Intensity-modulated arc therapy with dynamic multileaf collimation: an alternative to tomotherapy. Phys Med Biol 1995;40:1435-49.

4. Oliver M, Ansbacher W, Beckham WA. Comparing planning time, delivery time and plan quality for IMRT, RapidArc and Tomotherapy. J Appl Clin Med Phys 2009;10:117-31.

5. Pasler $M$, Wirtz H, Lutterbach J. Impact of gantry rotation time on plan quality and dosimetric verification-volumetric modulated arc therapy (VMAT) vs. intensity modulated radiotherapy (IMRT). Strahlenther Onkol 2011;187:812-9.

6. Tang J, Wang H. The radiotherapy of cervical cancer. Shanghai Medical \& Pharmaceutical Journal 2016;37:20-4.

7. Deng $\mathrm{H}$, Zhao $\mathrm{Y}$, Luo $\mathrm{W}$, et al. Research of postoperative patients with cervical cancer dosimetry of IMRT and VMAT radiotherapy technology. Chinese Journal of Cancer Prevention and Treatment 2017;24:708-13.

8. Tu J, Zuohelaguli M, Zhang J, et al. Comparison of dosimetry and toxicities between postoperative fixedfield intensity-modulated radiotherapy and image-guided radiation therapy/volumetric modulated arc therapy for cervical cancer. Chinese Journal of Radiation Oncology 2017;26:410-3.

9. Jones D. ICRU Report 50—Prescribing, Recording and Reporting Photon Beam Therapy. Med Phys 1994;21:833-4.

10. Kutcher GJ, Coia L, Gillin M, et al. Comprehensive QA for radiation oncology: report of AAPM Radiation Therapy Committee Task Group 40. Med Phys 1994;21:581-618.

11. Georg D, Stock M, Kroupa B, et al. Patient-specific IMRT verification using independent fluence-based dose calculation software: experimental benchmarking and initial clinical experience. Phys Med Biol 2007;52:4981-92.

12. Sellakumar P, Arun C, Sanjay SS, et al. Comparison of monitor units calculated by radiotherapy treatment planning system and an independent monitor unit verification software. Phys Med 2011;27:21-9.

13. Ma CM, Jiang SB, Pawlicki T, et al. A quality assurance phantom for IMRT dose verification. Phys Med Biol 2003;48:561-72.

14. Han Z, Ng SK, Bhagwat MS, et al. Evaluation of MatriXX for IMRT and VMAT dose verifications in peripheral dose regions. Med Phys 2010;37:3704-14.

15. Oulhouq Y, Rrhioua A, Zerfaoui M, et al. Dosimetric effect resulting from the collimator angle, the isocenter move, and the gantry angle errors. Iran J Med Phys 2019;16:355-61.

16. Heilemann G, Poppe B, Laub W. On the sensitivity of common gamma-index evaluation methods to MLC misalignments in Rapidarc quality assurance. Med Phys 
2013;40:031702.

17. Small W Jr, Bosch WR, Harkenrider MM, et al. NRG Oncology/RTOG Consensus Guidelines for delineation of clinical target volume for intensity modulated pelvic radiation therapy in postoperative treatment of endometrial and cervical cancer: an update. Int J Radiat Oncol Biol Phys 2021;109:413-24.

18. Taylor A, Rockall AG, Reznek RH, et al. Mapping pelvic lymph nodes: guidelines for delineation in intensitymodulated radiotherapy. Int J Radiat Oncol Biol Phys 2005;63:1604-12.

19. Ezzell GA, Burmeister JW, Dogan N, et al. IMRT commissioning: multiple institution planning and dosimetry comparisons, a report from AAPM Task Group 119. Med Phys 2009;36:5359-73.

20. Low DA, Harms WB, Mutic S, et al. A technique for the quantitative evaluation of dose distributions. Med Phys 1998;25:656-61.

21. Qin S, Zhang Y, Li H, et al. Effects of the setup errors

Cite this article as: Yuan Y, Chang XB, Hu LJ, Wang GQ, Xue YJ. Effect of isocenter deviation on volume modulated arc therapy plan results and gamma passing rate in the treatment of cervical cancer. Transl Cancer Res 2021;10(10):4403-4412. doi: 10.21037/ tcr-21-1514 on dose distribution of target area and organs at risk for prostate cancer. Chinese Journal of Radiation Oncology 2019;28:37-40.

22. Luo S, Wu H, He Z, et al. Development of methodology for quality audit of doses in target area and organ at risk and two dimensional dose distribution in intensity modulated radiotherapy. Chinese Journal of Radiological Medicine and Protection 2020;40:122-8.

23. Deng X, Huang S, Zhong N, et al. Dosimetry verification and quality assurance test of IMRT. Chinese Journal of Cancer 2001;20:1092-4.

24. Nelms BE, Zhen H, Tomé WA. Per-beam, planar IMRT QA passing rates do not predict clinically relevant patient dose errors. Med Phys 2011;38:1037-44.

25. Zhen H, Nelms BE, Tome WA. Moving from gamma passing rates to patient DVH-based QA metrics in pretreatment dose QA. Med Phys 2011;38:5477-89.

(English Language Editor: C. Betlazar-Maseh) 